\title{
Keyword Index Volume 11, 2006
}

\begin{tabular}{|c|c|c|c|c|c|}
\hline Adolescents & 50 & Early learning & 253 & Organizational commitment & 119 \\
\hline Adult identity & 25 & Education & 268 & Parental cognitions & 57 \\
\hline Age & 164,312 & Emerging adulthood & 25 & Parenting program & 57 \\
\hline Aggression & 79 & Emotions & 297 & Perceptual speed & 196 \\
\hline Aging & $96,304,312$ & Euro & 277 & Personality & 297 \\
\hline Aging stereotypes & 312 & Family & 268 & Planned behavior & 99 \\
\hline Agreement & 12 & Family preservation services & 57 & Positive psychology & 143 \\
\hline Albanian youth & 268 & Feminization & 1 & Postadolescence & 25 \\
\hline Anger & 297 & Figurative language & 128 & Posttraumatic stress disorder & 137 \\
\hline Anxiety & 297 & Foreign language acquisition & 289 & Practice & 164 \\
\hline Applied psychology & 149 & Frequently asked questions & 236 & Predictors of mortality & 224 \\
\hline Assessment & 297 & Future perspective & 149 & Professional associations & 149 \\
\hline Attitude formation & 99 & Gender & 268 & Professional ethics & 236 \\
\hline Blind people & 110 & Genotype & 143 & $\begin{array}{l}\text { Professional organizations }\end{array}$ & 236 \\
\hline Breaching of confidentiality & 236 & Group discourse & 57 & & \\
\hline Cancer & 182 & Group management & 57 & Prosocial behavior & 90 \\
\hline Categorization & 253,277 & Haptic object identification & 110 & Psychological development & 143 \\
\hline Chance model & 12 & Helping behavior & 90 & Psychological distress & 39 \\
\hline Child-rearing practices & 57 & Identity exploration and & & Psychology as a profession & 1 \\
\hline Children & 50 & commitment & 268 & Psychosocial risk & 57 \\
\hline Chronic health condition & 50 & Information seeking & 99 & Quality of life & , 312 \\
\hline Civil courage & 90 & Intention & 99 & Retraumatization & 137 \\
\hline Cognition & $96,224,304$ & Interpersonal stress & 39 & Role transitions & 25 \\
\hline Cognitive aging & 204 & Job involvement & 119 & Romania & 39 \\
\hline Cognitive decline & 172,182 & Joint analysis of longitudinal & & Scientific psychology & 149 \\
\hline Cognitive impairment & 312 & and survival models & 204 & Serotonin & 143 \\
\hline Cognitive plasticity & 312 & Карра & 12 & Social acknowledgment & 137 \\
\hline Collective efficacy & 312 & Kappa alternative & 12 & Social change & 25 \\
\hline Coping & 50 & Language proficiency & 289 & Social support & 39 \\
\hline Coronary heart disease & 182 & Life satisfaction & 312 & Sport & 143 \\
\hline Covariates & 12 & Longitudinal & 196 & Stroke & 182 \\
\hline Crime victims & 137 & Luxembourg & $\begin{array}{r}149 \\
70\end{array}$ & Structural equation modeling & 204 \\
\hline $\begin{array}{l}\text { Curiosity } \\
\text { Death }\end{array}$ & $\begin{array}{l}297 \\
224\end{array}$ & Marginal deviations & $\begin{array}{r}79 \\
137\end{array}$ & Survival analysis & 204 \\
\hline $\begin{array}{l}\text { Death } \\
\text { Decision-making }\end{array}$ & $\begin{array}{l}224 \\
236\end{array}$ & $\begin{array}{l}\text { Media report } \\
\text { Memory }\end{array}$ & $\begin{array}{l}131 \\
304\end{array}$ & Swimming & 143 \\
\hline $\begin{array}{l}\text { Decision-making } \\
\text { Dementia }\end{array}$ & $\begin{array}{l}236 \\
172\end{array}$ & $\begin{array}{l}\text { Memory } \\
\text { Money }\end{array}$ & 277 & Tactile pictures & 110 \\
\hline Depression & 297 & Moral norms & 99 & Terminal decline $172,196,204$ & 224 \\
\hline Development & 79 & Mortality & 2,182 & Text comprehension & 128 \\
\hline Development of figurative & & Multilevel modeling & 224 & University of Luxembourg & 149 \\
\hline competence & 128 & Neurodegenerative diseases & 304 & Utrecht Work Engagement Scale & 119 \\
\hline Disagreement & 12 & Object and social knowledge & 253 & Well-being & 312 \\
\hline Discriminant validity & 119 & Occupation & 268 & Women in psychology & 1 \\
\hline Dropout & 164 & Optimal aging & 312 & Working memory & 289 \\
\hline
\end{tabular}

